\title{
Polimedia y grupos públicos: un estudio comparado sobre los procesos de selección de plataformas digitales
}

\author{
Mercedes Durá Lizán | mdura@umh.es \\ Universidad Miguel Hernández \\ Raúl Travé Molero | rtrave@umh.es \\ Universidad Miguel Hernández \\ Antonio Miguel Nogués-Pedregal | amnogues@umh.es \\ Universidad Miguel Hernández
}

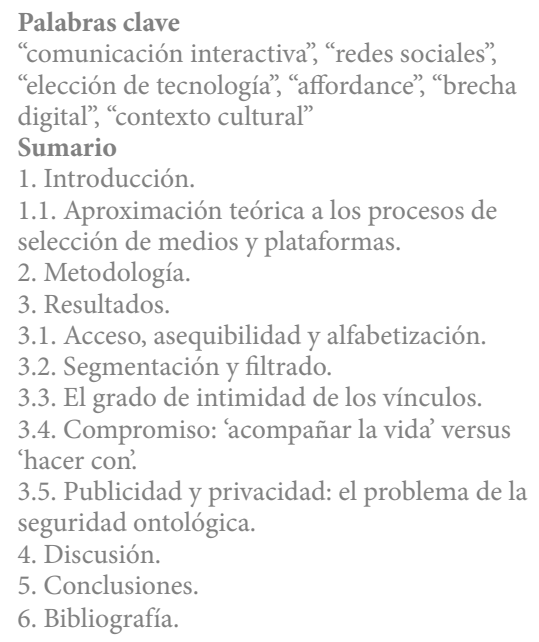

En nuestros contextos de investigación, los grupos públicos digitales han adquirido un lugar predominante en la comunicación pública local. Sin embargo, para este fin sus habitantes han optado por diferentes entornos: Facebook en Santa Pola (España) y WhatsApp en Itacaré (Brasil). En este artículo exponemos los factores económicos y socioculturales asociados a dicha elección, siguiendo los planteamientos de la Teoría Polimedia (Madianou \& Miller, 2013). Nuestros resultados han sido extraídos a partir de una etnografía comparada de corte cualitativo sobre comunicación pública $\mathrm{y}$ herramientas digitales. Concluimos que a priori el motivo de estas preferencias está relacionado con el acceso, la asequibilidad y la alfabetización, pero además interfieren otros elementos como los mecanismos de filtrado o la intimidad, privacidad y el sentido de compromiso asociado a las plataformas. Se trata de una aproximación novedosa en la literatura científica en español a las prácticas de selección entre plataformas, que puede servir como punto de partida para futuras investigaciones centradas en los usos de la tecnología.

\section{Cómo citar este texto:}

Mercedes Durá Lizán, Raúl Travé Molero, Antonio Miguel Nogués-Pedregal (2021): Polimedia y grupos públicos: un estudio comparado sobre los procesos de selección de plataformas digitales, en Miguel Hernández Communication Journal, Vol. 12 (1), pp. 223 a 242. Universidad Miguel Hernández, UMH (Elche-Alicante). DOI: https://doi. org/10.21134/mhcj.v12i.353 


\section{Polymedia and public groups: a comparative study on the selection process of digital platforms}

Mercedes Durá Lizán |mdura@umh.es

Universidad Miguel Hernández

Raúl Travé Molero | rtrave@umh.es

Universidad Miguel Hernández

Antonio Miguel Nogués-Pedregal | amnogues@umh.es

Universidad Miguel Hernández

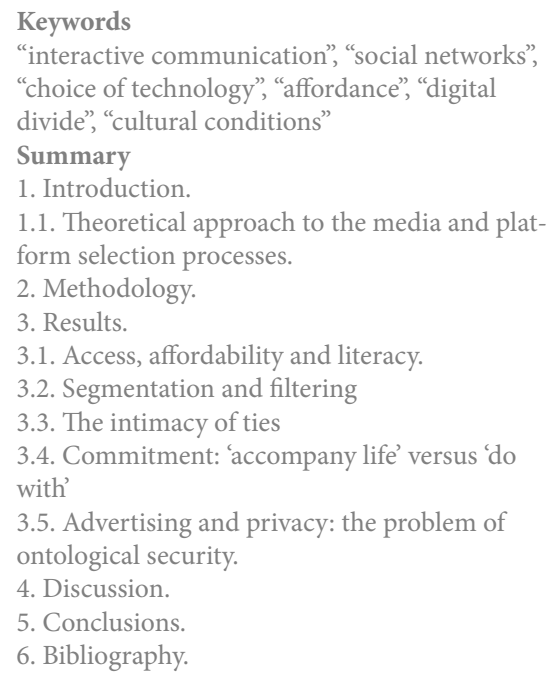

\section{Abstract}

Digital public groups have acquired a predominant place in local public communication in our research contexts. However, for this purpose, the population has chosen different technological environments: Facebook in Santa Pola (Spain) and WhatsApp in Itacaré (Brazil). In this article, we expose the economic and socio-cultural factors associated with this choice, following the approaches of the Polymedia Theory (Madianou and Miller, 2013). Our results have been extracted from a comparative qualitative ethnography on public communication and digital tools. We conclude that the reasons for these preferences are, at first, access, affordability and literacy, but also interfere other elements, like filtering mechanisms, privacy, intimacy of ties and sense of commitment associated with Platforms. This is a novel approach in Spanish scientific literature for multiplatform selection practices between platforms, that can be usefull as starting point for future research focused on the technological uses.

\section{How to cite this text:}

Mercedes Durá Lizán, Raúl Travé Molero, Antonio Miguel Nogués-Pedregal (2021): Polimedia y grupos públicos: un estudio comparado sobre los procesos de selección de plataformas digitales, en Miguel Hernández Communication Journal, Vol. 12 (1), pp. 223 a 242. Universidad Miguel Hernández, UMH (Elche-Alicante). DOI: https://doi. org/10.21134/mhcj.v12i.353 


\section{Introducción}

Los grupos creados en plataformas digitales se han convertido en espacios privilegiados de la comunicación pública en contextos locales (Graham, 2015; Gregory, 2015; Swart, Peters, \& Broersma, 2019), como hemos podido confirmar en los municipios donde hemos realizado nuestra investigación, Itacaré en Brasil y Santa Pola en España. Sin embargo, sus poblaciones han optado por diferentes entornos para este fin: los itacaerenses han creado sus grupos públicos en la aplicación de mensajería instantánea WhatsApp, mientras que los santapoleros se han decantado por la red social Facebook. Esta elección coincide con la que se ha producido en otros municipios de ambos países, por lo que podemos pensar que los resultados a los que hemos llegado a partir de nuestra etnografía son extrapolables a otros contextos.

Nuestro artículo expone algunos factores que intervienen en la selección de una determinada tecnología por un grupo social amplio y parte de la hipótesis de que algunas características del contexto socioeconómico y cultural influyen en este proceso. Dicha afirmación deriva de una etnografía comparada transnacional, realizada en dos municipios que son también dos destinos turísticos de primer orden, Itacaré y Santa Pola. Esta etnografía planteó también la hipótesis de que la mediación fundamental del contexto -especialmente la acción estructurante del turismo (Nogués-Pedregal, 2020)-influye en el modo en que las prácticas de comunicación digital se insertan en los circuitos comunicativos locales. Asimismo, nos planteábamos que esta mediación debía influir en las preferencias entre plataformas.

Ambos contextos presentan algunas características comunes, como su tamaño -en torno a los 30.000 habitantes- la tradición pesquera y la posición periférica en sus respectivas regiones. Sin embargo, estos municipios presentan una diferencia en su desarrollo económico y humano muy significativa, ya que Santa Pola tiene una renta bruta media per cápita de 22.354 euros (Agencia Estatal de Administración Tributaria [AEAT], 2017) y forma parte de lo que en el actual orden mundial se considera primer mundo, mientras que Itacaré está localizada en el Nordeste brasileño, una de las regiones más humildes del país y su PIB apenas representa los 9.333,22 R \$ per cápita -1.496,41€- (Instituto Brasileiro de Geografia e Estadística [IBGE], 2010). El nivel educativo representa el mismo desequilibrio: según los datos del último censo de Itacaré el 58,86\% de la población no tenían la educación básica completa (IBGE, 2010), mientras que en Santa Pola tan solo el 9,34\% no tiene ningún tipo de estudio (Instituto Nacional de Estadística [INE], 2011).

La transición hacia el modelo turístico ha seguido también caminos diferentes. Santa Pola es un destino maduro que ha sufrido un crecimiento urbanístico intensivo y expansivo para sustentar un modelo de turismo residencial, mientras que Itacaré es un destino de corta estancia en crecimiento, que dispone de un gran territorio rural y bastante población dispersa. Como consecuencia de estos modelos diferentes, Santa Pola ha recibido un movimiento migratorio de personas mayores basado en el consumo, mientras que Itacaré ha recibido, sobre todo, inmigrantes económicos de la región. De hecho, en este último municipio las personas de otras regiones del país y extranjeros suponían apenas el 5,16\% de la población censada en 2010 (IBGE, 2010), mientras que en Santa Pola el 44,39\% de sus residentes provenían de otras comunidades o países (INE, 2011). 


\subsection{Aproximación teórica a los procesos de selección de medios y plataformas}

La proliferación de plataformas y aplicaciones y la complejidad de los fenómenos de transmedialidad y convergencia (Jenkins, 2008), han renovado el interés por los procesos de elección de tecnologías concretas para las interacciones interpersonales o grupales mediadas. Algunos de los planteamientos desarrollados en torno a la mediatización (Braga, 2006; Hepp \& Hasebrink, 2015) aportan una dimensión amplia desde la que observar el modo en que las tecnologías intervienen en las interacciones sociales, que puede ayudar a explicar algunas preferencias.

Del mismo modo, los desarrollos planteados desde la mediación (Couldry, 2004) y la domesticación (Ardévol \& Pinyol, 2009; Haddon, 2016; Matassi, Boczkowski, \& Mitchelstein, 2019; Silverstone, 1993; Wong, 2010), facilitan la comprensión de los procesos socioculturales que llevan a los sujetos a adoptar una tecnología, dotarla de un significado y darle un espacio en la vida doméstica o cotidiana (Ardévol \& Pinyol, 2009; Haddon, 2016; Matassi et al., 2019; Morley, 2003; Sandoval \& Cabello, 2019; Silverstone, 1993; Wong, 2010).

Desde la dimensión de la cultura material, Daniel Miller y Don Slater (2000: 7-15) destacaron algunas dinámicas desde las que pensar los procesos de apropiación de internet, que se generan a partir de la tensión entre las formas de vida y las potencialidades de la tecnología: la de objetivación, la de mediación, la de libertad normativa y la de posicionamiento. La visión relacional de algunos planteamientos contemporáneos de la Ecología de los medios también resulta interesante para comprender los diferentes usos (Ito et al., 2009; Scolari, 2015; Strate, 2004), entendidos como la integración de las características de un sistema general técnico, social, cultural y basado en el lugar, en el que los componentes no son descomponibles o separables (Horst, Herr-Stephenson, \& Robinson, 2018: 31).

Un concepto operativo para plantearnos los procesos de selección es el de "media repertoires" (repertorios de medios) formulado por Uwe Hasebrink y Hanna Domeyer (2012) y entendido como la totalidad de los medios o plataformas que usa regularmente un individuo o grupo social. Estos autores plantean que las relaciones entre los diferentes elementos de este repertorio tienen una coherencia interna y establecen una colección de parámetros bastante amplia que guían esta composición, como las estrategias de autorepresentación, los sistemas de relaciones, la movilidad de los actores, la integración en las rutinas cotidianas, la relevancia, la diversidad, la complementeriedad funcional y la compatibilidad/competencia entre los componentes, el prestigio social asociado a los medios o plataformas o la forma en que encajan en el proyecto de vida personal de los individuos (Hasebrink \& Domeyer, 2012: 759). Nick Couldry (2016) planteó también esta nueva realidad en que los individuos tienen que aprender a gestionar una colección de medios realizando una selección pragmática de múltiples opciones.

Para aproximarnos al modo en que las potencialidades de una tecnología influyen en su elección o descarte, resultan interesantes las aportaciones de los estudios de las affordance, concepto que se sitúa a medio camino entre las propiedades incrustadas en la tecnología 
y la lectura que de ella hacen sus usuarios (Davis \& Chouinard, 2016; Evans, Pearce, et al., 2017) destacan una serie de potencialidades percibidas: anonimato del usuario, persistencia y visibilidad de la información, intimidad percibida y posibilidades de colaboración con otros. Según Jenny Davis y James Chouinard (2018: 2-6) las propiedades de la tecnología influyen en las conductas finales, al solicitar, exigir, permitir, alentar, desalentar y rechazar algunas prácticas, pero insisten en destacar que el ambiente social y cultural es determinante en la percepción, la destreza y la legitimidad de cada tecnología.

José van Dijck (2016) analiza cómo las potenciales de las arquitecturas moldean los significados que los usuarios construyen en sus interacciones, considerando la tecnología un espacio de diálogo entre empresas productoras y consumidores. En su misma línea, Michael Bossetta (2018) describe la arquitectura de cuatro plataformas -Facebook, Twitter, Instagram y Snapchat- y destaca que la estructura de la red, la funcionalidad, el filtrado algorítmico y el modelo de datos median en su objeto de investigación, la comunicación política. Por su parte, Ariel Urevich (2016) analiza el manejo del tiempo realizado por Facebook, biográfico y retrospectivo, frente a la volatilidad de aplicaciones de mensajería instantánea.

Mirca Madianou y Daniel Miller (2013: 172) formulan la que denominan Teoría Polimedia, a la que describen como "una estructura integrada de posibilidades" que los usuarios explotan para gestionar sus relaciones emocionales y afectivas. Según estos autores, una vez que se dan algunas condiciones fundamentales, como son el acceso, la asequibilidad y la alfabetización, la elección de uno u otro medio recae sobre aspectos morales, sociales y emocionales introduciendo la dimensión cultural como elemento central en este proceso (Madianou \& Miller, 2013: 171). Estos investigadores se apoyan en trabajos previos, entre ellos los de Baym, Broadbent y Gershon.

Nancy K. Baym, Yan Bing Zhang y Mei Chen Lin (2004: 7), destacan una serie de parámetros -interactividad, estructura temporal, señales sociales, almacenamiento, replicabilidad, alcance y movilidad- que pueden ser utilizados para comparar productivamente diferentes medios entre sí, y que influyen en los modos en que las personas los usan para sustentar sus relaciones sociales.

Por su parte Stefana Broadbent y Valerie Bauwens (2008) estudian qué tipo de medios son considerados apropiados para las interacciones entre vínculos fuertes y cuáles se usan cuando los individuos se plantean ampliar las conexiones de vínculos débiles. Broadbent (2012) destaca criterios como la privacidad, la discreción, la necesidad de retroalimentación inmediata, la disponibilidad del interlocutor, la frecuencia de conversación, la familiaridad o formalidad de la relación, el tiempo disponible y la calidad del intercambio (cit. en Madianou \& Miller, 2013: 173). Por su parte, Ilana Gershon (2010) describe las valoraciones morales que hacen sus alumnos sobre el uso de determinadas herramientas digitales para prácticas relacionales concretas como dejar una relación.

Janine Bargas y Rousiley Maia (2017: 40) consideran el carácter conversacional de WhatsApp un factor fundamental para los grupos de activistas políticos, junto a la inmediatez, la menor exposición del perfil personal, así como la simplicidad de la interfaz y 
la compresión de archivos que acelera los intercambios. Por su parte Maria L. Calero (2014: 107) destaca la imposibilidad de controlar la recepción en los mensajes en WhatsApp como una característica que genera cierto rechazo entre sus usuarios.

También se han investigado las posibilidades que el teléfono móvil y las aplicaciones de mensajería ofrecen para la coordinación y la movilidad de los usuarios (Ling \& Lai, 2016; Ling \& Yttri, 2002). Siguiendo esta idea Kenton O'Hara, Richard Harper, et al. (2014) consideran que WhatsApp es una herramienta clave para la coordinación funcional y afectiva con aquellos con los que se comparte la vida.

Patrick J. Bateman, Jacqueline C. Pike y Brian S. Butler (2011) concluyen en su investigación que la percepción de publicidad en las redes sociales como Facebook o Instagram frena la "autorevelación" que los usuarios hacen de sus gustos o ideas. Por su parte Bernie Hogan (2010) detectó que los métodos de agregación de contendidos de las plataformas, así como los mecanismos de acceso, convierten a algunas redes sociales como Facebook en espacios de exhibición -"exhibition spaces"-. Danah Boyd y Alice Marwick (2011) consideran que la actuación de los adolescentes en las redes sociales está condicionada por su forma de entender los regímenes públicos y privados de sus vidas.

\section{Metodología}

Este artículo es resultado de una etnografía comparada sobre la producción de significados a través de las interacciones públicas digitales. En el marco de esta investigación hemos indagado sobre las prácticas de selección de plataformas a fin de conocer los argumentos o razonamientos que los actores hacen de sus preferencias.

La aproximación etnográfica permite abordar de forma contextualizada las prácticas digitales, evitando atribuirles causalidades arbitrarias (Hine, 2004: 186; Miller \& Slater, 2000: 3). Asimismo, el abordaje comparado permite, por un lado, detectar los particularismos locales y las formas propias de los habitantes de un territorio de estar en internet y, por otro, evitar generalizaciones erróneas (Madianou \& Miller, 2013: 184).

El trabajo de campo se ha desarrollado desde septiembre a diciembre de 2017 y de septiembre de 2018 a febrero de 2019 en Itacaré, y entre abril y junio de 2018 y entre junio y septiembre de 2019 en Santa Pola. Las técnicas empleadas han sido las entrevistas y observación participante. En Itacaré se han desarrollado un total de 87 entrevistas y en Santa Pola un total de 41 entrevistas de duración variada, aproximadamente entre los 30 y los 120 minutos. La muestra fue seleccionada de forma aleatoria y no probabilística, tratando que fuera compensada, es decir, que existiera una amplia representación sociodemográfica.

Mientras que en Santa Pola las entrevistas tuvieron lugar en establecimientos, espacios públicos o en lugares de trabajo, en Itacaré varias de ellas fueron realizadas en los propios hogares de los entrevistados, debido a que resultan especialmente accesibles al tratarse generalmente de plantas bajas, orientadas a la calle, por lo que la vida trascurre, en gran medida, en los portales. Este apunte etnográfico refleja el modo en que la configuración del 
espacio urbano favorece un determinado modelo de interacción social.

Paralelamente, nos integramos en varios grupos digitales considerados públicos por los entrevistados de ambos contextos, lo que nos ha permitido observar de primera mano las prácticas de los miembros y triangular los resultados de las entrevistas. En total hemos formado parte de 16 grupos de Facebook de Santa Pola y de 6 grupos de WhatsApp en Itacaré, prestando especial atención a un grupo de cada municipio: Eres de Santa Pola si... en Santa Pola e Itacaré Urgente en Itacaré.

\section{Resultados}

En general a los entrevistados les cuesta formular las razones de sus actos. Sin embargo, tras una breve reflexión, emiten algunas explicaciones. Otras, sin embargo, las hemos extraído a partir de su narración o de la observación prolongada de sus prácticas. Factores económicos, educativos, económicos, tecnológicos, sociales y culturales han dado lugar a que WhatsApp y Facebook se institucionalicen como los canales para hablar de los asuntos públicos en el ámbito local.

\subsection{Acceso, asequibilidad y alfabetización}

El acceso a internet en nuestros contextos viene determinado por la configuración espacial y urbanística. Dada la extensión territorial de Itacaré, no tiene cobertura de red en muchas zonas rurales. En Santa Pola, en las áreas construidas para albergar segundas residencias -en las que con el tiempo se ha ido asentando población permanente- el servicio suele ser deficitario, según expresan sus residentes, debido a la orografía de estas áreas residenciales que se extienden sobre la sierra y sobre una lengua de arena entre las salinas y el mar.

El factor económico es especialmente significativo al comparar estos contextos, ya que para la mayor parte de santapoleros resulta asequible disponer de un ordenador personal y de una tarifa plana de internet. No sucede lo mismo en Itacaré, debido a la coincidencia de un bajo nivel adquisitivo y de los altos precios de la tecnología. Así lo confirmaba un estudio de los precios promedio de catorce productos tecnológicos sobre un total de 71 países (Línio, 2016), en el que Brasil se posiciona en el número 69, mientras que España ocupa la posición número 13 .

Sin embargo, la mayor parte de habitantes de Itacaré pueden acceder a un dispositivo móvil, ya sea nuevo o de segunda mano. De hecho, en el último censo del municipio (IBGE, 2010) se computaban 3.914 hogares con teléfono celular, mientras que tan solo 820 hogares disponían de ordenador y, de estos, solo 575 estaban conectados a internet.

A esto se suma que muchas personas de Itacaré disponen de tarjetas de prepago en su dispositivo. Ante esta circunstancia sus habitantes recurren a varias estrategias, como usar prioritariamente la aplicación WhatsApp, que gracias a su bajo consumo alarga la duración de los datos, o conectarse cuando tienen ocasión desde redes WIFI. Asimismo, desde 2018, la operadora con más cobertura en el municipio, ofrece el recurso de intercambiar mensajes 
y archivos en WhatsApp sin descontar datos del crédito disponible en sus tarifas prepago.

Una vez que tienen acceso a un dispositivo, WhatsApp ha permitido a la mayor parte de población de Itacaré tener por primera vez contacto con internet, frente a otros recursos que resultan demasiado complejos para buena parte de la población. Como destaca un vecino: "muitas pessoas aqui não sabem mexer com Facebook". Así pues, la facilidad de uso de esta aplicación -que permite comunicarse incluso a aquellos que no saben escribir, mediante el envío de audios y el reconocimiento de voz- ha permitido incorporar a muchos de los anteriormente considerados analfabetos digitales a internet. Es el caso de un morador de Santo Antônio -uno de los barrios más humildes de la ciudad- de 67 años, que no fue a la escuela y se dedica a la venta ambulante, y cuenta que usa la herramienta, sobre todo, para comunicarse a través de un grupo con su familia que reside en una ciudad cercana. Sin embargo, asegura que no se conecta a internet porque no sabe entrar, lo que demuestra que no es consciente de estar conectado a la red cuando se comunica con sus seres queridos.

$\mathrm{Al}$ igual que en el caso anterior una vecina del Porto de Tras de 37 años, se conectó por primera vez a WhatsApp sin haber usado nunca un ordenador, gracias a que sus hijas le instalaron la aplicación en el móvil: "eu peço muito que elas façam as coisas pra mim, porque eu sou assim uma pessoa que não guenta [sic] ver (risos)...”. Esta anécdota muestra como las redes de relaciones próximas -familiares, amigos, compañeros de clase o de trabajo- atraen a los que están fuera hacia sus redes virtuales a fin de tenerlos accesibles a través de su teléfono móvil (ver Wong, 2010: 181).

En Santa Pola, la población más joven empezó a usar Facebook desde sus ordenadores personales a partir del año 2009, cuando se democratizó la plataforma en España. Posteriormente, y atraídas en parte por el éxito de los grupos, algunas personas mayores han iniciado un proceso de autoaprendizaje, como señala la creadora del grupo con mayor número de miembros en Santa Pola: "empezó la gente así pues ponle de treinta y algo, más o menos como yo cuando lo abrí, y luego ya un montón de gente mayor enseguida". Una vecina de 74 años que ha vivido casi toda su vida en Santa Pola confirma esta idea y explica que los hijos les enseñan y que además aprenden "trasteando". Esto anima a pensar la relación entre vejez y tecnología en Santa Pola como un proceso especialmente activo de domesticación.

\subsection{Segmentación y filtrado}

Uno de los motivos que indican varios vecinos de Itacaré para decantarse por WhatsApp es la velocidad con que los mensajes llegan a otros contactos: "WhatsApp é mais instantâneo, mais dinâmico, então você consegue isso com muita facilidade. Até pra você filtrar o que seja bom e ruim, ele é mais rápido". Por el contrario, perciben que el filtrado algorítmico de Facebook reduce la velocidad y autonomía en la selección de la información, así como la inmediatez en la recepción.

Los habitantes de Itacaré realizan un pre-filtrado, como señala una vecina de un barrio tradicional de la ciudad: "Alguns eu fico, outros me colocam, eu vou saindo, mas alguns, os 
mais interessantes, que você vê que tem mais informações, as informações até melhores, a gente vai ficando". Además, una vez dentro, muchas personas siguen filtrando la información persona a persona, simplemente no prestando atención a los mensajes de aquellos que no resultan de su interés, como señala el creador de uno de los grupos: "as minhas postagens me identificam. Então, quem não gosta de... da minha linha de pensamento não abre as minhas mensagens, não acessa”.

Por el contrario, al sugerir al creador del primer grupo de Facebook de Santa Pola si no había pensado en la posibilidad de usar WhatsApp, respondía: “eso es una locura!". Crear un espacio en la aplicación de mensajería para tratar temas de índole municipal le parece disfuncional, por la cantidad de mensajes que se acumulan en la aplicación y la visualización instantánea que la caracteriza. Frente a ello, Facebook privilegia para él lo relevante y disminuye la tensión ante la disyuntiva generada por el deseo de, por una parte, estar conectado y, por otra, tener una cierta tranquilidad que garantice una calidad de vida aceptable.

\subsection{El grado de intimidad de los vínculos}

Hasta finales del siglo pasado, en que se asfaltó la carretera de acceso, Itacaré permanecía relativamente aislada. Además, el tamaño pequeño del núcleo urbano y un estilo de vida que trascurre principalmente en la calle está ayudando a preservar los vínculos estrechos de la población. "Itacaré é um copo" o "Em Itacaré todo o mundo é familia", son algunas de las expresiones usadas por sus habitantes para definir los estrechos lazos de sus habitantes. Por este motivo una nativa que forma parte de la junta diretiva del Sindicato de Trabalhadores Públicos señala: "Facebook é para outra coisa! Para pautas mais grandes, regionais ou estaduais... Para coisas daqui, locais, é melhor o WhatsApp”. En Itacaré, WhatsApp permite reunir un aforo limitado de personas, que es considerado adecuado para discutir cuestiones que se entiende que incumben solo a sus habitantes.

A pesar de que Santa Pola conserva ciertos rasgos de relación de una antigua villa de pescadores, la urbanización y el crecimiento de la población que han generado la inmigración y el turismo residencial, junto a las trasformaciones en los estilos de vida y en el paisaje de la ciudad, han dado lugar a que hayan surgido vínculos que se parecen más a los característicos de la vida urbana que a los de las comunidades tradicionales (Bauman, 2000). En este contexto, Facebook permite mantener cierta distancia con el resto de participantes y responde así a los requisitos asociados al espacio público, mientras que WhatsApp es sentido como parte del espacio íntimo, como explica una vecina de 49 años natural de Santa Pola: "es como más personal y hay que tener ahí los teléfonos y no te apetece tampoco que tengan datos tuyos personales".

\subsection{Compromiso: 'acompañar la vida' versus 'hacer con'}

Facebook, es sentido por las personas con las que hemos conversado en Itacaré como un medio para "acompanhar a vida", es decir, intercambiar retazos de la propia existencia 
con las personas con las que no se mantiene necesariamente un contacto cotidiano, sobre todo en este contexto fuertemente marcado por la movilidad de su población: "Eu acho o Facebook interessante assim, pra você contatar pessoas que você não vê faz tempo, que você gosta, mas por motivos outros estão aí pelo mundo, que nem os seus amigos... Então você... mas de uma maneira ou outra você acompanha a vida".

En esta línea el propietario de una agencia turística señala que cada vez entra menos a esta plataforma porque no tiene tiempo de "ficar olhando". Por el contrario, WhatsApp le permite obtener de forma instantánea la información que necesita conocer del entorno, a partir de la experiencia de los que lo cohabitan:

Também não tenho tempo de entrar mais. Já vai chegando a idade que eu vejo, de vez em quando, não tem nada pra fazer, eu vejo. Tô cansado, não quero ficar programado. Não quero fazer alguma coisa, aí eu entro e vejo. Mas geralmente eu não tô mais olhando. Dificilmente eu olho. Eu olho mais no WhatsApp, que a mensagem mais rápida que chega é no WhatsApp. Se alguém tomou... aconteceu alguma coisa, aquele cara, na Passagem [barrio de la ciudad], mesmo eu não estando no grupo, já divulgam e rapidinho chega aqui, né. Menos de um minuto já tá em todos os grupos.

Precisamente por este tipo de contacto leve que propicia Facebook, que exige un nivel de compromiso y de confianza menor, es frecuente que algunos de sus habitantes se relacionen a través de la plataforma con personas que no conocen y con las que solo comparten cierta afinidad. Frente a esta apertura asociada a Facebook, WhatsApp es un espacio para lo conocido, para la red de relaciones del entorno caracterizada por rasgos como la intimidad, la profundidad y la autenticidad como explica un moto-taxista de la ciudad:

No Facebook é essa onda aí, que a gente faz amizade com gente que a gente nunca nem viu! [...] Eu prefiro o WhatsApp, que o WhatsApp é mais íntimo... O WhatsApp é o grupo de amigo meu, é o grupo de moto-taxi, é um grupo de minha família, é amigo meu...

En los grupos públicos funciona esta misma lógica de coordinación y confianza, ya que están integrados por personas con las que se interactúa diariamente, como explica otro taxista de la ciudad: "a maioria conheço do dia a dia, são pessoas do dia a dia [énfasis en la entonación]".

También en Santa Pola la aplicación WhatsApp es vista como un espacio cerrado, orientado a las relaciones personales y a la coordinación cotidiana, solo que en este caso no se encuentra cabida en esta categoría para cualquier persona de la ciudad. Así, una vecina nacida en Santa Pola cuenta que en WhatsApp participa en grupos "de amigas, compañeras de trabajo, madres del cole..." mientras que en Facebook está "en algo de Eres de santa Pola..., cosas de estas, compra-venta Santa Pola...". 


\subsection{Publicidad y privacidad: el problema de la seguridad ontológica}

Esta apertura asociada a Facebook provoca que esta red social sea percibida en Itacaré como un entorno inseguro. Por ello un nativo de la ciudad, explica la razón de ser de los grupos de WhatsApp del siguiente modo: "Se eu posto no Facebook, é o Brasil inteiro que vai ver. E no Instagram, pior ainda. Porque até pra fora do Brasil o povo vai saber".

Por el contrario, los grupos de WhatsApp son percibidos como lugares cerrados, capaces de contener la información que no se desea que trascienda hacia el exterior, como explica el administrador de uno de ellos:

O nosso interesse é mais local, então a gente consegue filtrar para os interesses da localidade. O Facebook não, a gente vai espalhar, pra muita gente, pra outros países, então... o nosso interesse não é esse, não é chamar a atenção de quem tá lá fora. Nosso interesse é orgânico, é pra questão local, pras pessoas que estão aqui.

Este enunciado demuestra la conciencia permanente de que el contenido y la forma en que se comunican en la red va a afectar al posicionamiento global del territorio, contribuyendo a forjar la imagen de destino turístico del municipio. Usando WhatsApp para los asuntos locales, los habitantes de Itacaré controlan el alcance del contenido que difunden, del mismo modo que cuando quieren que algo trascienda tienden a compartirlo en Facebook.

Para los habitantes de Santa Pola, Facebook también es una red orientada a la publicidad. "Todo lo pueden ver ahí, es que lo tienen todo o casi todo", señala uno de los administradores. Por este motivo la plataforma ha albergado en este municipio una serie de prácticas comunicativas basadas en la difusión de información, que tradicionalmente usaban otros soportes como los medios locales o los muros y paredes del pueblo. En Itacaré la divulgación de información de interés para la comunidad se realiza en los grupos de WhatsApp y, aquella que se considera que se considera de interés para los posibles visitantes, en las páginas creadas para ello en Facebook. De este modo, los itacaerenses también separan lo privado -el espacio de la comunidad local-, de lo público -el espacio para los turistas o personas de otros lugares-.

A los habitantes de Santa Pola también les preocupa que esta apertura y publicidad afecte a la imagen de la ciudad, sobre todo, debido a la presencia de veraneantes en los grupos. Uno de los administradores comenta que este es uno de los motivos por los que han aumentado las reglas y restringido la admisión de nuevos miembros solo a aquellos que expliciten algún tipo de vínculo con el pueblo. Ante las críticas vertidas por otros vecinos ante este aumento del control, explica que "miro por mi pueblo y miro por la gente que viene de afuera".

\section{Discusión}

El uso de Facebook en Santa Pola y de WhatsApp en Itacaré para hablar de los asuntos públicos de ambos municipios es parte de la "conciencia práctica" de sus habitantes, es decir, de aquello que "los actores saben tácitamente sobre el modo de 'ser con' en contextos 
de vida social sin ser capaces de darles una expresión discursiva directa" (Giddens, 2011: 24). Resulta muy complicado para los actores definir estos conocimientos primarios, experimentados por ellos como objetivos (Berger \& Luckmann, 1937: 36).

Por ello, cuando les cuestionamos por los motivos de usar estas aplicaciones sus reacciones eran de desconcierto, como si no comprendieran siquiera que se pudiera formular una pregunta tan fuera de lo común (Garfinkel, 1967: 36). Además, a esto hay que añadir que no existe una única respuesta, ya que los usos asignados a la tecnología son en primera instancia coyunturales, es decir, resultado de una serie de circunstancias materiales y sociales específicas (Davis \& Chouinard, 2016).

Como destacan Mirca Madianou y Daniel Miller (2013: 175-176) los requisitos que influyen a priori en que las personas se decanten por una tecnología u otra son el acceso, la asequibilidad y la alfabetización. En Santa Pola, donde la población tiene un mayor nivel económico y adquisitivo, una parte pionera de la población empezó a usar Facebook desde su ordenador personal y, a remolque de esto, se incorporaron otros estratos sociales.

Para la población de Itacaré la convergencia del dispositivo móvil y de WhatsApp ha proporcionado una opción económica y fácil para conectarse por primera vez a internet (Gómez Cruz \& Harindranath, 2020: 3), lo que supone que bastantes usuarios pasen de emplear el teléfono móvil solo para hacer llamadas, a explorar las opciones de conectividad (ver Silva, 2010: 191-192).

Se confirma en este contexto la idea planteada por Castells, Mireia Fernández-Ardevol, et al. (2007: 377), de que la tecnología móvil ha pasado de ser un lujo de los países desarrollados a convertirse en un artefacto de consumo de masas, que permite que los países y regiones en vías de desarrollo reduzcan la brecha de la conectividad. Aun así, es evidente la existencia de una brecha digital que presenta múltiples dimensiones (Guilló \& Mancebo-Aracil, 2017: 418) y que influye, más que en el acceso, en los modos en que se accede a la red y los usos que se hacen de la tecnología (Alva de la Selva, 2015: 274-279; Madianou \& Miller, 2013: 176).

Una vez sorteadas estas barreras básicas, las formas en que los diferentes medios o plataformas cubren sus necesidades relacionales y afectivas son fundamentales para su elección (Madianou \& Miller, 2013: 171). Así pues, hemos detectado que los mecanismos de filtrado, el tipo de vínculos sociales que encontramos en cada contexto, el sentido de compromiso asociado a las plataformas, así como la separación de los ámbitos de la vida percibidos como públicos o privados son claves para estas elecciones.

Como señalan Castells et al. (2007: 121), las diferencias más significativas en la incorporación de algunas tecnologías se encuentran al comparar distintos países y se ha relacionado con determinados rasgos de las culturas nacionales. "Se ha sugerido que la población de culturas sociables y colectivas acepta ciertos comportamientos de uso que no serían aceptados en sociedades más individualistas" (Castells, Fernández-Ardevol, Qiu, \& Sey, 2007: 122). Así, por una parte, América del Sur, con una cultura colectivista, una gran distancia al poder y mayor prevención de la incertidumbre, y, por otra, Europa Occidental, con culturas individualistas y una distancia baja al poder, habrían desarrollado patrones diferenciados en 
la adopción de la tecnología móvil (cit. en Castells et al., 2007: 123).

Coincidiendo con esta idea, algunos itacaerenses muestran una gran tolerancia a la saturación informativa que implica el alto volumen de mensajes que son recibidos a través de los grupos de WhatsApp (Calero, 2014: 93) y destacan la instantaneidad de la herramienta WhatsApp que les permite reproducir "las condiciones naturales de la conversación presencial” (Bargas \& Maia, 2017: 40). Podemos afirmar que la posibilidad de seleccionar o personalizar voluntariamente a qué grupos de WhatsApp desean pertenecer y decidir a quién van a enviar cada información supone para los itacaerenses una victoria de la voluntad personal frente al dominio del algoritmo de Facebook (ver Castells et al., 2007: 181).

Por el contrario, los santapoleros evitan estar en plataformas o aplicaciones que les consuman tiempo y requieran de una alta implicación o esfuerzo personal. La plataforma Facebook les permite mantener una distancia con las informaciones compartidas por otros miembros de los grupos (Bateman et al., 2011: 79; Couldry \& van Dijck, 2015: 6) al priorizar las informaciones relevantes (Urevich, 2016: 226).

Estas preferencias diferentes están relacionadas también con la configuración local de la comunicación, resultante de las características culturales de ambos contextos. Así pues, el tamaño de la población urbana y los diferentes tipos de agregación social que predominan en uno y otro territorio tienen asociados niveles de compromiso diferentes, lo que influye en que sus miembros se decanten por una u otra plataforma. En ambas poblaciones la aplicación WhatsApp está reservada para la coordinación cotidiana (Ling \& Yttri, 2002) y para las relaciones íntimas o personales (Urevich, 2016: 233), pero cambia el alcance de esta categorización de los vínculos en ambos contextos.

La población de Itacaré mantiene lazos cercanos a la idea de comunidad (Maya, 2004), un sentimiento de pertenencia y una cultura en común (Krause Jacob, 2001). Por ello consideran la red social Facebook más apropiado para las relaciones de afinidad (Castells, 2001: 147, 2003: 11) y WhatsApp adecuado para las 'relaciones-nosotros' (cit. en Ritzer, 1993: 381) o 'relaciones hacer' (O’Hara et al., 2014) caracterizadas por la interacción cotidiana y una mayor intimidad (cit. en Boellstorff, 2016).

En Santa Pola los vínculos comunicativos se han diluido y sus habitantes se conciben cada vez más a ellos mismos como 'colectividad' (Delgado, 2007). Facebook es percibida como una plataforma abierta y global (Urevich, 2016; Van Dijck, 2016) y por tanto más apropiada para la comunicación de carácter público. Aunque la apertura de Facebook es, en un principio, contradictoria con la idea de privacidad (Bossetta, 2018; Urevich, 2016; Van Dijck, 2016), separar en diferentes plataformas o entornos los regímenes públicos y privados genera cierta tranquilidad a sus habitantes (Boyd \& Marwick, 2011). Los grupos de Facebook, debido a que admiten a un número elevado de miembros y que no es necesario conocer personalmente a los administradores para acceder a ellos, potencian además la conexión de vínculos débiles (Broadbent \& Bauwens, 2008), lo que permite acoger también a la población creciente del municipio y a los turistas vacacionales. 
De este modo, la percepción de WhatsApp como un entorno instantáneo, íntimo y seguro (Davis \& Chouinard, 2016: 5) motiva a los habitantes de Itacaré a emplearlo para mantener la conversación sobre algunos asuntos polémicos en la privacidad de la comunidad, y evitar así dañar la imagen de la ciudad de cara al exterior, habida cuenta de que los entornos digitales resultan fuentes fundamentales de información turística (Andrade Suárez, 2012). Por otra parte, el carácter público de Facebook (Bateman et al., 2011; Ellison, Steinfield, \& Lampe, 2007) facilita a los santapoleros poner una distancia entre ese ámbito y el privado y, al mismo tiempo, paliar las carencias locales de espacios de información pública (ver Swart et al., 2018: 7), así como autoafirmar su identidad tradicional en el espacio global (Miller \& Slater, 2000: 13; Van Dijck, 2016: 150). Además, este proceso de producción y mantenimiento de la identidad territorial resulta favorecido por la temporalidad cronológica y retrospectiva de Facebook (Urevich, 2016; Van Dijck, 2016).

Esta selección diferenciada de las plataformas, en función de los binomios seguridad/ inseguridad y privacidad/apertura, muestra que la construcción digital de la imagen de la ciudad, mediante un manejo adecuado de las impresiones, es sentida como una responsabilidad pactada y compartida (Goffman, 1959: 88-116, 223-226). Sin embargo, si los retazos de informaciones accesibles en la red proyectan una imagen decisiva del destino en la mente del turista de Itacaré, no tiene una repercusión similar en la imagen que los residentes temporales de Santa Pola tienen sobre el pueblo, porque el vínculo del veraneante con el territorio no se construye a partir internet, sino que se basa en la experiencia propia y en sus vínculos emocionales y relacionales. Y, para algunos de ellos, formar parte de los grupos de Facebook es, incluso, un modo de fortalecer su arraigo al lugar.

\section{Conclusiones}

Estudiar los procesos de adopción de la tecnología de forma diacrónica o, como es nuestro caso, comparando diferentes contextos, permite observar cómo influyen las características de estos lugares y evitar generalizaciones o asociaciones causales arbitrarias (Madianou \&

Miller, 2013; Miller \& Slater, 2000).

Los usos que los sujetos hacen de las plataformas y aplicaciones sociales derivan del encuentro de las propiedades de la tecnología y de las necesidades y posibilidades de quienes las consumen. En un primer momento, la elección de una tecnología entre otras está influenciada por la pervivencia de una brecha digital, que ya no se puede valorar tanto en términos de acceso sino, más frecuentemente, en relación a factores económicos o educativos. Una vez superada esta barrera, son claves las lecturas relacionales y emocionales de elementos como los mecanismos de filtrado o sensación de privacidad, la intimidad de los vínculos y el sentido de compromiso asociado a las plataformas.

\section{Bibliografía}

Agencia Estatal de Administración Tributaria, A. (2017). Renta bruta media per cápita Santa Pola. www.epdata.es. Recuperado de https://www.epdata.es/datos/datos-graficos- 
estadisticas-municipio/52/santa-pola/6691

Alva de la Selva, A. R. (2015). Los nuevos rostros de la desigualdad en el siglo XXI: la brecha digital. Revista Mexicana de Ciencias Políticas y Sociales, 60(223), 265-285. https:// doi.org/10.1016/s0185-1918(15)72138-0

Andrade Suárez, J. M. (2012). El uso de Internet como fuente de información turística: propuesta metodológica para el análisis de su importancia en la creación de la imagen del destino. Papers de Turisme, (52), 44-62.

Ardévol, E., \& Pinyol, E. (2009). La domesticación de Internet. Recuperado de https// www. photographicsocialvision.org/domestic/pdf/elisenda_ardevol_y_eva_pinyol_cast. pdf

Bargas, J. de K. R., \& Maia, R. C. M. (2017). Quilombolas no WhatsApp: o papel do aprendizado coletivo nas lutas por reconhecimento. Comunicação Mídia e Consumo, 14(41), 31. https://doi.org/10.18568/cmc.v14i41.1391

Bateman, P. J., Pike, J. C., \& Butler, B. S. (2011). To disclose or not: Publicness in social networking sites. Information Technology and People, 24(1), 78-100. https://doi. org/10.1108/09593841111109431

Bauman, Z. (2000). Modernidad líquida. Buenos Aires: Fondo de la Cultura Económica Argentina.

Baym, N. K., Zhang, Y. B., \& Lin, M. C. (2004). Social interactions across media: Interpersonal communication on the internet, telephone and face-to-face. New Media and Society, 6(3), 299-318. https://doi.org/10.1177/1461444804041438

Berger, P., \& Luckmann, T. (1937). La Construcción Social De La Realidad. Buenos Aires: Amorrortu Editores.

Boellstorff, T. (2016). For Whom the Ontology Turns: Theorizing the Digital Real. Current Anthropology, 57(4), 387-407. https://doi.org/10.1086/687362

Bossetta, M. (2018). The Digital Architectures of Social Media: Comparing Political Campaigning on Facebook, Twitter, Instagram, and Snapchat in the 2016 U.S. Election. Journalism and Mass Communication Quarterly, 95(2), 471-496. https://doi. org/10.1177/1077699018763307

Boyd, D., \& Marwick, A. E. (2011). Social Privacy in Networked Publics: Teens' Attitudes, Practices, and Strategies. A Decade in Internet Time: Symposium on the Dynamics of the Internet and Society, September 2011, (September 2009), 1-29. https://doi. org/10.1037/0003-066X.63.2.111

Braga, J. L. (2006). Mediatização como processo interacional de referência. Animus Revista Interamericana de Comunicação Midiática, 2(julho/dez), 9-35. 
Broadbent, S. (2012). Approaches to Personal Communication. Digital Anthropology, 127145.

Broadbent, S., \& Bauwens, V. (2008). Feature: Understanding convergence. Interactions, 15(1), 23. https://doi.org/10.1145/1330526.1330536

Calero, M. L. (2014). El Discurso del Whatsapp: entre el Messenger y el SMS. Oralia, 17, 85-114.

Castells, M. (2000). Internet y la sociedad red. En U. O. de Catalunya (Ed.), Conferencia de Presentación del Programa de Doctorado sobre la Sociedad de la Información y el Conocimiento. (pp. 1-13).

— (2003). La Galaxia Internet. Barcelona: Plaza \& Janés Editores.

Castells, M., Fernández-Ardevol, M., Qiu, J. L., \& Sey, A. (2007). Comunicación móvil y sociedad, una perspectiva global. Madrid: Ariel.

Couldry, N. (2004). Theorising media as practice. Social Semiotics, 14(2), 115-132. https:// doi.org/10.1080/1035033042000238295

- (2016). Life with the media manifold: Between freedom and subjection. En L. Kramp, N. Carpentier, A. Hepp, R. Kilborn, R. Kunelius, H. Nieminen, ... S. Toson (Eds.), Politics, Civil Society and Participation: Media and Communications in a Transforming Environment (pp. 25-39). Bremen: Edition Lumière.

Couldry, N., \& van Dijck, J. (2015). Researching Social Media as if the Social Mattered. Social Media and Society, 1(2). https://doi.org/10.1177/2056305115604174

Davis, J. L., \& Chouinard, J. B. (2016). Theorizing Affordances: From Request to Refuse. Bulletin of Science, Technology \& Society, 36(4), 241-248. https://doi. org/10.1177/0270467617714944

Ellison, N. B., Steinfield, C., \& Lampe, C. (2007). The benefits of facebook «friends»: Social capital and college students' use of online social network sites. Journal of Computer-Mediated Communication, 12(4), 1143-1168. https://doi.org/10.1111/j.10836101.2007.00367.x

Evans, S. K., Pearce, K. E., Vitak, J., \& Treem, J. W. (2017). Explicating Affordances: A Conceptual Framework for Understanding Affordances in Communication Research. Journal of Computer-Mediated Communication, 22(1), 35-52. https://doi.org/10.1111/ jcc4.12180

Garfinkel, H. (1967). Studies in Ethnomethododology. New Jersey: Prentice-Hall.

Gershon, I. (2010). The Breakup 2.0 Disconnecting over New Media. Ithaca and London: Cornell University Press. 
Giddens, A. (2011). La constitución de la sociedad. Bases para la teoría de la estructuración (2a). Buenos Aires: Amorrortu.

Goffman, E. (1994). La representación de la persona en la vida cotidiana (2a). Buenos Aires: Amorrortu.

Gómez Cruz, E., \& Harindranath, R. (2020). WhatsApp as 'technology of life': Reframing research agendas. First Monday, 25(1-6), 1-15. https://doi.org/http://dx.doi.org/10.5210/ fm.v25i1.10405

Graham, T. (2015). Everyday political talk in the internet-based public sphere. En S. Coleman \& D. Freelon (Eds.), Handbook of Digital Politics. Cheltenham, UK: Edward Elga. https://doi.org/10.13140/RG.2.1.1217.5524

Gregory, J. (2015). Connecting with the past through social media: The Beautiful buildings and cool places Perth has lost Facebook group. International Journal of Heritage Studies, 21(1), 22-45. https://doi.org/10.1080/13527258.2014.884015

Guilló, M., \& Mancebo-Aracil, J. F. (2017). Comunicación y participación online: la evolución de los procesos participativos en entornos virtuales. Miguel Hernández Communication Journal, 8, 413-434.

Haddon, L. (2016). Análisis de la domesticación y estudio sobre el uso que hace la población infantil de los Smartphones y las Tablets. Revista de Estudios de Juventud, (111), 141-153.

Hasebrink, U., \& Domeyer, H. (2012). Media repertoires as patterns of behaviour and as meaningful practices: A multimethod approach to media use in converging media environments. Participations. Journal of Audience \& Reception Studies, 9(2), 757-779.

Hepp, A., \& Hasebrink, U. (2015). Interação humana e configurações comunicativas: transformações culturais e sociedades midiatizadas. Parágrafo, 2(3), 75-89.

Hine, C. (2004). Etnografía virtual. Barcelona: Editorial UOC.

Hogan, B. (2010). The Presentation of Self in the Age of Social Media: Distinguishing Performances and Exhibitions Online. Bulletin of Science, Technology \& Society, 30(6), 377-386. https://doi.org/10.1177/0270467610385893

Horst, H. A., Herr-Stephenson, B., \& Robinson, L. (2010). Media Ecologies. (M. Ito, Ed.), Hanging Out, Messing Around, and Geeking Out: kids living and learning with new media. Cambridge: The MIT Press. https://doi.org/10.7551/mitpress/8402.001.0001

Instituto Brasileiro de Geografia e Estatística [IBGE]. (2010). Censo demográfico Itacaré. Recuperado de https://cidades.ibge.gov.br/brasil/ba/itacare/ pesquisa $/ 23 / 22469$ ?detalhes $=$ true

Instituto Nacional de Estadística INE. (2011). Características censales de Santa Pola. Recuperado de http://www.argos.gva.es/

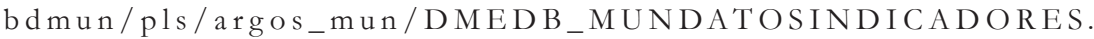


DibujaPagina? aNMunId $=3121 \&$ aNIndicador $=2 \&$ aVLengua $=\mathrm{c}$

Ito, M., Horst, H., Bittanti, M., Boyd, D., Herr-Stephenson, B., Lange, P. G., ... Robinson, L. (2009). Living and Learning with New Media: Summary of Findings from the Digital Youth Project. Cambridge: The MIT Press.

Jenkins, H. (2008). Convergence Culture La cultura de la convergencia de los medias de comunicación. Barcelona: Paidós.

Krause Jacob, M. (2001). Hacia una Redefinición del Concepto de Comunidad -cuatro ejes para un análisis crítico y una propuesta-. Revista de Psicología, X(2), 49-60.

Ling, R., \& Lai, C.-H. (2016). Microcoordination 2.0: Social Coordination in the Age of Smartphones and Messaging Apps. Journal of Communication, 66(5), 834-856. https:// doi.org/10.1111/jcom.12251

Ling, R., \& Yttri, B. (2002). Hyper-coordination via mobile phones in Norway. En J. Katz \& M. Aakhus (Eds.), Perpetual contact: Mobile communication, private talk, public performance. (Vol. 30/99, pp. 1-27). Cambridge: Cambridge University Press.

Línio. (2016). Índice de precios de la tecnología. Recuperado de https://www.linio.com.pe/ sp/indice-precios-tecnologia-2016

Madianou, M., \& Miller, D. (2013). Polymedia: Towards a new theory of digital media in interpersonal communication. International Journal of Cultural Studies, 16(2), 169-187. https://doi.org/10.1177/1367877912452486

Matassi, M., Boczkowski, P. J., \& Mitchelstein, E. (2019). Domesticating WhatsApp: Family, friends, work, and study in everyday communication. New Media \& Society, 21(10), 21832200. https://doi.org/10.1177/1461444819841890

Maya, I. (2004). Sentido de comunidad y potenciación comunitaria. Apuntes de Psicología, 22(2), 187-211.

Miller, D., \& Slater, D. (2000). The internet. An Ethnographic Approach. Oxford: Berg.

Morley, D. (2003). What's 'home' Got to do with it?: Contradictory Dynamics in the Domestication of Technology and the Dislocation of Domesticity. European Journal of Cultural Studies, 6(4), 435-458. https://doi.org/10.1177/13675494030064001

Nogués-Pedregal, A. M. (2020). El turismo como contexto. Disparidades. Revista de Antropología, 75(1), 001. https://doi.org/10.3989/dra.2020.001c

O’Hara, K. P., Massimi, M., Harper, R., Rubens, S., \& Morris, J. (2014). Everyday dwelling with WhatsApp. En Proceedings of the 17th ACM conference on Computer supported cooperative work \& social computing - CSCW '14 (Vol. 15, pp. 1131-1143). New York, New York, USA: ACM Press. https://doi.org/10.1145/2531602.2531679

Ritzer, G. (1993). Teoría sociológica clásica. Madrid: McGraw-Hill. 
Sandoval, L. R., \& Cabello, R. (2019). Límites a la domesticación: usos obligados, reticencia y austeridad en la apropiación de la telefonía móvil. Dixit, (31), 82-99. https://doi. org/10.22235/d.vi31.1849

Scolari, C. A. (2015). Los ecos de McLuhan: ecología de los medios, semiótica e interfaces. Palabra Clave - Revista de Comunicación, 18(4), 1023-1056. https://doi.org/10.5294/ pacla.2015.18.4.4

Silva, S. R. (2010). Estar no tempo, estar no mundo: a vida social dos telefones celulares em un grupo popular (Tesis inédita de doctorado). Universidade Federal de Santa Catarina, Florianópolis.

Silverstone, R. (1993). Domesticating the revolution: information and communication technologies and everyday life. Aslib Proceedings, 45(9), 227-233. https://doi.org/10.1108/ eb051328

Strate, L. (2004). A media ecology review. Communication Research Trends, 23(3), 3-48.

Swart, J., Peters, C., \& Broersma, M. (2019). Sharing and Discussing News in Private Social Media Groups. Digital Journalism, 7(2), 187-205. https://doi.org/10.1080/21670811.201 8.1465351

Urevich, A. (2016). El tiempo todo en Facebook. Aposta, 69(abril-junio), 217-238.

Van Dijck, J. (2016). La cultura de la conectividad: una historia crítica de las redes sociales. Buenos Aires: Siglo XXI Editores.

Wong, C. Y. (2010). Limitaciones y alcances del enfoque de domesticación de la tecnología en el estudio del teléfono celular. Comunicación y sociedad, 13, 173-200. 
MHCJ Vol. 12 (1) | Año 2021 - Artículo no 11 (178) - Páginas 223 a 242 - mhjournal.org

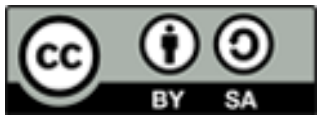

Licencia Creative Commons

Miguel Hernández Communication Journal

mhjournal.org

\section{Cómo citar este texto:}

Mercedes Durá Lizán, Raúl Travé Molero, Antonio Miguel Nogués-Pedregal (2021): Polimedia y grupos públicos: un estudio comparado sobre los procesos de selección de plataformas digitales, en Miguel Hernández Communication Journal, Vol. 12 (1), pp. 223 a 242. Universidad Miguel Hernández, UMH (Elche-Alicante). DOI: https://doi. org/10.21134/mhcj.v12i.353 